\title{
Therapeutic Radiology
}

National Cancer Institute

\section{Source}

National Cancer Institute. Therapeutic Radiology. NCI Thesaurus. Code C17759.

The medical specialty concerned with the use of electromagnetic or particulate radiation in the treatment of disease. 